\title{
Nano-oxidation of silicon surfaces by noncontact atomic-force microscopy: Size dependence on voltage and pulse duration
}

\author{
Montserrat Calleja and Ricardo García ${ }^{a)}$ \\ Consejo Superior de Investigacione Cientificas, Instituto de Microelectrónica de Madrid, C/Isaac Newton 8, \\ 28760 Tres Cantos, Madrid, Spain
}

(Received 24 January 2000; accepted for publication 12 April 2000)

\begin{abstract}
Local oxidation of silicon surfaces by noncontact atomic-force microscopy is an emerging and promising method for patterning surfaces at the nanometer scale due to its very precise control of the feature size. Here, we study the voltage and pulse duration conditions to generate a motive of a given height with the minimum lateral size. We find that for a fixed tip-sample separation, the combination of short pulses and relatively high voltages $(\sim 20 \mathrm{~V})$ produces the highest height:width ratio. The application of relatively high voltages produces a fast growth rate in the vertical direction while the lateral diffusion of oxyanions is inhibited for short pulses. The above results are applied to generate lines of tens of microns in length with an average width at half maximum of about 10 nm. (C) 2000 American Institute of Physics. [S0003-6951(00)04823-3]
\end{abstract}

Local oxidation of semiconductor and metallic surfaces by scanning-probe microscopy is arguably the most reliable and versatile scanning-probe-based lithography method for the fabrication of nanometer-scale structures. A conducting tip is brought into contact or near contact with a surface that is wetted by a water layer. An external voltage pulse is applied between the tip (negative electrode) and the sample to induce the anodic oxidation of the surface. Silicon, ${ }^{1}$ III-V semiconductors, ${ }^{2}$ titanium, ${ }^{3}$ and molybdenum ${ }^{4}$ surfaces have been locally oxidized by tunneling and atomic-force microscopy (AFM). The fabrication of single-electron ${ }^{5}$ and metal-oxide ${ }^{6}$ transistors illustrates some of the nanoelectronic applications. Local oxidation in combination with laser lithography is also useful to fabricate nanomechanical devices. ${ }^{7}$ Machining of silicon structures has also been demonstrated by AFM oxidation. ${ }^{8}$ Furthermore, the local oxidation is compatible with the operation of parallel-tip arrays. ${ }^{9}$ This opens the possibility to pattern $\mathrm{cm}^{2}$ surfaces with nanometer-size motives.

To gain a precise control of the feature size and to improve the reproducibility of the whole process, we have proposed the use of noncontact AFM to locally oxidize surfaces. ${ }^{10,11}$ This method requires the field-induced formation of a liquid bridge. The liquid bridge provides the oxyanions $\left(\mathrm{OH}^{-}, \mathrm{O}^{-}\right)$needed to form the oxide. It also confines the lateral extension of the region to be oxidized. This approach has dramatically improved the tip's lifetime for modification purposes. For example, it has been shown that arrays of several thousands of dots with a periodicity of $40 \mathrm{~nm}$ and an average width of $10 \mathrm{~nm}$ can be generated. ${ }^{11}$

A nanolithography method based on noncontact AFM oxidation should also demonstrate the ability to make long, uniform, narrow, and continuous lines. Lines and dots are the basic elements to generate complex nanostructures. Furthermore, lines could be used as etch masks in the fabrication of nanowires. ${ }^{12}$ However, to generate in a reproducible and

\footnotetext{
a) Author to whom correspondence should be addressed; electronic mail: rgarcia@imm.cnm.csic.es
}

controlled way very small features, the relative role of the different parameters that control the oxide size in noncontact AFM should also be clarified.

Here, we study the oxide width and height dependence on voltage and pulse duration for noncontact AFM oxidations. Those studies provide the conditions to pattern a motive of a given width and height. The results are applied to pattern continuous lines that are several microns in length while their lateral width is about $10 \mathrm{~nm}$, i.e., they show a 1000:1 ratio.

Several experiments have already outlined the influence of the oxidation time on the oxide height. ${ }^{10,13,14}$ It has also been shown that the doping in silicon samples ${ }^{15,16}$ influences the reaction kinetics. Additionally, some experiments have shown a voltage modulation dependence. ${ }^{17,18}$ The voltage modulation has been linked to a space-charge buildup process. ${ }^{16}$ Recently, we have shown that the liquid bridge size also influences the lateral dimensions of the oxide. ${ }^{11}$ Although different models have already been proposed to explain the mechanism and kinetics of the local oxidation in silicon, ${ }^{13,14,16,19}$ the rich and complex interdependence of the above parameters has yet to be explained.

The experiments were performed with an atomic-force microscope operated in noncontact mode (Nanoscope III, Digital Instruments) with additional circuits to perform the oxidation. The microscope was placed into a closed box with inlets for dry and $\mathrm{H}_{2} \mathrm{O}$-saturated nitrogen. The relative humidity was kept around 30\%-60\%. Doped $n^{+}$-type silicon cantilevers were used (Nanosensors, Germany). The average force constant $\left(k_{c}\right)$ and resonance frequency $\left(f_{0}\right)$ were about $34 \mathrm{~N} / \mathrm{m}$ and $330 \mathrm{kHz}$, respectively. The cantilever was excited at its resonance frequency. The samples were $p$-type $\mathrm{Si}(100)$ with a resistivity of $14 \Omega \mathrm{cm}$. Due to exposure to air, the surface has a native-oxide layer of about $2 \mathrm{~nm}$. The feedback is switched off during the lithographic process. Further description of the dynamic parameters needed to operate the AFM in a noncontact mode can be found elsewhere. ${ }^{20}$

The process to locally oxidize silicon surfaces in noncontact AFM has three major steps. ${ }^{11}$ First, an oscillating tip 


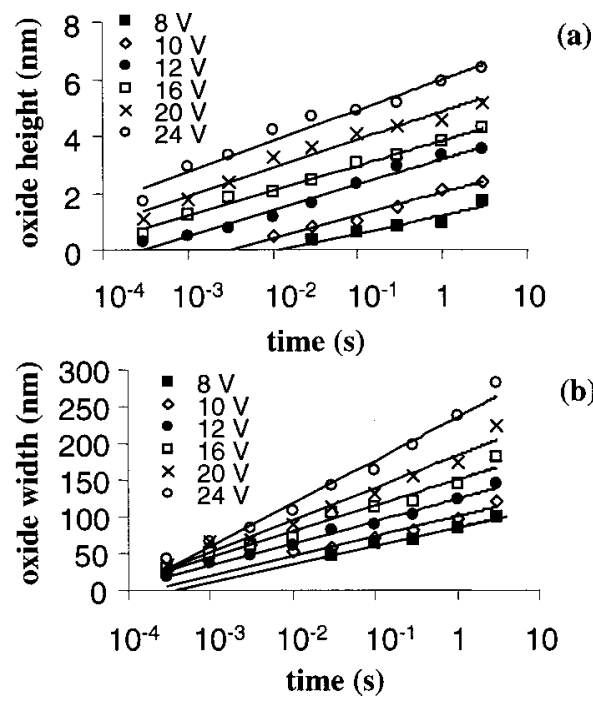

FIG. 1. Oxide size dependence on applied voltage and pulse duration. (a) Height dependence on pulse duration for different voltages. (b) Apparent width dependence on pulse duration for different voltages. Before the application of a voltage pulse, the average tip-sample separation and the oscillation amplitude were, respectively, 7 and $5 \mathrm{~nm}$.

is placed $5-10 \mathrm{~nm}$ above the surface, then a voltage pulse is applied to form a liquid bridge between the tip and sample. Third, another voltage is applied to induce the anodic oxidation. We have also shown that the oxide width decreases by increasing the average tip-sample separation. However, thinning the liquid bridge is somehow limited to a few nanometers. Once the liquid bridge is formed, it can only be stretched by $4-10 \mathrm{~nm}$ before it breaks.

To determine the conditions to produce a feature of a given size, the tip is placed at a fixed separation above the sample surface (average value). Then, we apply different voltage pulses. The data allow us to study the dependence of the lateral and vertical dimensions of the resulting oxide dots as a function of voltage and pulse duration.

Figures 1(a) and 1(b) show the dot's size dependence on pulse duration for five pulses of $8,10,12,16,20$, and $24 \mathrm{~V}$, respectively. Both width and height show a logarithmic dependence on the pulse duration. The logarithmic dependence of the oxide height on time has been reported previously, ${ }^{10,13,14}$ although a detailed model to explain it is still lacking. For a given pulse duration the width and height increase with the applied voltage. Unless otherwise stated, the oxide width refers to the apparent dot's width as measured from an AFM image. Due to tip-sample convolution effects, the real oxide width of the dot could be several times smaller than its apparent width. ${ }^{11}$ The slope of the oxide width curves also depends on the voltage [Fig. 1(b)]. This reflects the variation of the electrical field along the base of the liquid bridge.

Empirical height and width dependencies on time and voltage can be deduced from Fig. 1:

$$
\begin{aligned}
& h=h_{0}(V)+h_{1}(V) \ln t, \\
& w=w_{0}(V)+w_{1}(V) \ln t,
\end{aligned}
$$

where

$$
h_{0}=-2.1+0.5 \mathrm{~V}-0.006 \mathrm{~V}^{2}
$$

Downloaded 26 Feb 2010 to 161.111.180.191. Redistribution subject to AlP license or copyright; see http://apl.aip.org/apl/copyright.jsp

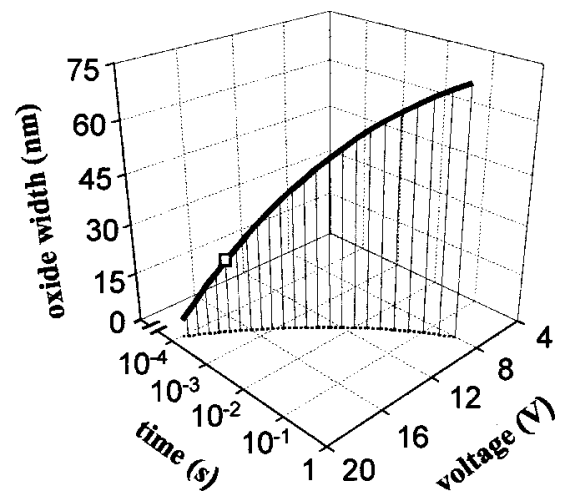

FIG. 2. Apparent oxide width dependence on applied voltage and pulse duration for an oxide mark of a given height, $h=1 \mathrm{~nm}$. The vertical lines are shown as a guide to the eye. The square represents the shortest experimental pulse used in these experiments.

and

$$
\begin{aligned}
& h_{1}=0.1+0.03 \mathrm{~V}-0.0005 \mathrm{~V}^{2}, \\
& w_{0}=11.6+9 \mathrm{~V} \text { and } w_{1}=2.7+0.9 \mathrm{~V} .
\end{aligned}
$$

In the above equations size, voltage, and time are expressed in nanometers, volts, and seconds, respectively.

Figures 1(a) and 1(b) indicate that the combination of short pulses and small voltages produces the smallest marks. However, the above result is an obvious conclusion that underlines the electrochemical character of the anodic oxidation. More important is to realize that for any practical purpose a mark must have a minimum thickness. For example, to block the formation of PtSi films a minimum thickness of $1 \mathrm{~nm}$ is required. ${ }^{21}$ Then, the growth of an oxide should incorporate the restriction of having a given height.

From Eqs. (1)-(4) it is possible to derive the coordinates $(V, t)$ that generate a mark of a height ranging between 0.5 and $5 \mathrm{~nm}$. The curve that contains the $(V, t)$ points that produce a dot of $1 \mathrm{~nm}$ in height is shown in Fig. 2. Figure 2 also shows that an increase of the voltage requires a decrease of the pulse duration to keep the height at $1 \mathrm{~nm}$. In other words, different combinations of short pulses at high voltages or long pulses at relatively low voltages could be applied to generate a dot of a given height. However, the experimental dot with the minimum width is obtained for the pulse that combines the shortest duration $(0.3 \mathrm{~ms})$ with a high voltage $(17 \mathrm{~V})$. This value $(0.3 \mathrm{~ms})$ was the shortest voltage pulse that could be generated by our instrument. The extrapolation of the curve to shorter pulses indicates that a dot's width of 5 $\mathrm{nm}$ could be obtained for a pulse of $0.1 \mathrm{~ms}$ at $20 \mathrm{~V}$.

The above result can be qualitatively understood as follows. The electrolysis of water molecules within the liquid bridge produces the oxyanions $\left(\mathrm{OH}^{-}, \mathrm{O}^{-}\right)$. Initially, the electrical field drives the oxyanions towards the silicon interface. It also effectively confines the oxyanions within a cylinder of diameter of the neck of the bridge. However, an increase of the pulse duration favors the thermal diffusion of ionic species in the $\mathrm{SiO}_{x}$ /liquid bridge interface. It is assumed that the diameter of the base of the liquid bridge is several times larger than its neck.

The lateral diffusion implies an increase of the oxide width. Assuming a diffusion constant of $\mathrm{OH}^{-}$in the solidliquid interface ${ }^{22}$ of about $D=10^{-9} \mathrm{~cm}^{2} \mathrm{~s}^{-1}$, then the aver- 


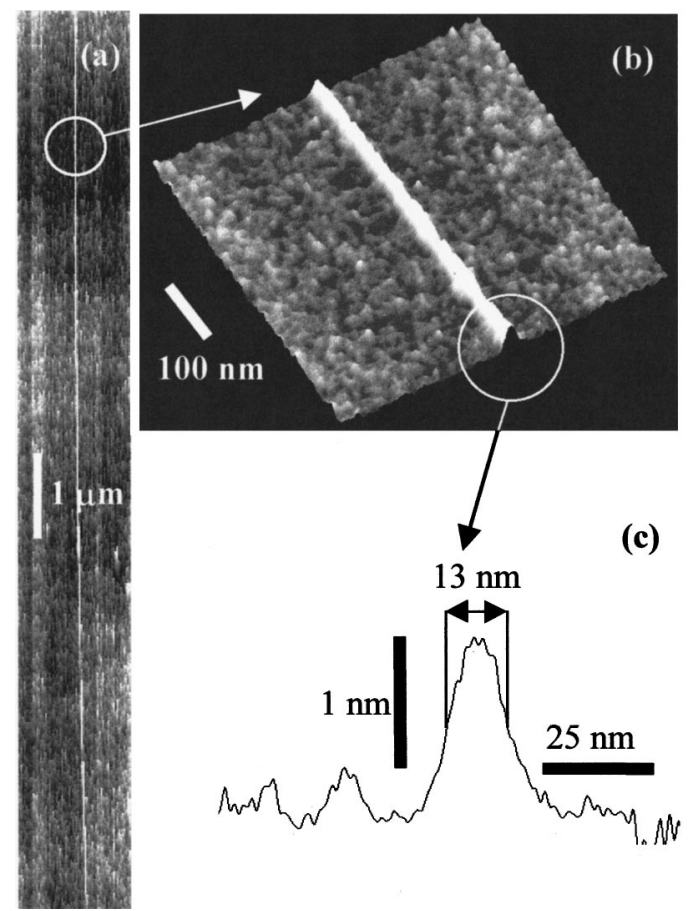

FIG. 3. AFM image of an oxide line made with the noncontact AFM method. (a) Overall aspect. (b) Three-dimensional section of the line shown in (a). (c) Cross section (apparent value) along the arrow shown in (b). Average tip-sample separation, $11 \mathrm{~nm}$.

age distance traveled by an ion is given by $r \sim(4 D t)^{1 / 2}$. For $t=10^{-4} \mathrm{~s}$, the above expression gives $r \sim 6 \mathrm{~nm}$. We believe that this effect should apply for oxidation times, say below $10^{-1} \mathrm{~s}$. Those times already imply average distances of about $200 \mathrm{~nm}$, i.e., larger than the experimental dot width, even though the measured width overestimates the real lateral size of the dots. For longer pulses, other factors such as the lateral dependence of the electric field at the $\mathrm{SiO}_{x}$ /liquid bridge interface and the space-charge buildup should dominate the growth rate. On the other hand, a high-voltage pulse is needed to have a fast growth rate in the vertical direction to reach the fixed height.

We have applied the above results to generate long and narrow lines with sub-1-nm tolerances. Contact AFM oxidation has produced lines of up to $5 \mu \mathrm{m}$ long. However, in most cases the lines are either broad or show width variations of several $\mathrm{nm}$. Broad features are related to a large water meniscus while width variations could be attributed to changes in the tip-sample separation. To avoid those problems, we have chosen to build a line as a sequence of single dots. Each dot is generated by the application of a single pulse. Between pulses the feedback is switched on to keep a fixed tip-sample separation (average value). At the same time the tip is laterally displaced.

Figure 3 shows an $11-\mu \mathrm{m}$-long line with a full width at half maximum of $13 \mathrm{~nm}$. The line is a sequence of $2 \times 10^{4}$ pulses of $20 \mathrm{~V}$ for $1 \mathrm{~ms}$. Between pulses the tip is laterally displaced $0.5 \mathrm{~nm}$. The small number of data points in the $y$ direction (256) and the length of the line give rise to the pixeled aspect of the image. A three-dimensional section of the line is shown in Fig. 3(b). The cross section [Fig. 3(c)] allows us to measure the height and width of the line.

In summary, we have found that for a fixed tip-sample separation, the application of short pulses at high voltages produces the highest height:width ratio in the fabrication of oxide marks by noncontact AFM. It is also predicted that a dot $1 \mathrm{~nm}$ in height and $5 \mathrm{~nm}$ in width could be generated by the application of a voltage pulse of $0.1 \mathrm{~ms}$ at $20 \mathrm{~V}$. The application of short pulses restricts the lateral diffusion of ionic species while a high-voltage pulse produces a fast growth rate in the $z$ direction. We have applied those findings to generate uniform and continuous $11-\mu \mathrm{m}$-long lines with a full width at half maximum of $13 \mathrm{~nm}$.

The authors acknowledge fruitful discussions with John Dagata and Francesc Pérez-Murano. This work was financially supported by the Commission of the European Communities, ESPRIT Contract No. 22955 (LASMEDS) and the Comunidad Autónoma de Madrid.

${ }^{1}$ J. A. Dagata, J. Schneir, H. H. Harary, C. J. Evans, M. T. Postek, and J. Bennet, Appl. Phys. Lett. 56, 2001 (1990).

${ }^{2}$ R. Held, T. Vancura, T. Heinzel, K. Ensslin, M. Holland, and W. Wegscheider, Appl. Phys. Lett. 73, 262 (1998).

${ }^{3}$ H. Sugimura, T. Uchida, N. Kitamura, and H. Masuhara, Appl. Phys. Lett. 63, 1288 (1993).

${ }^{4}$ J. Shirakashi, M. Ishii, K. Matsumoto, N. Miura, and M. Konagai, Jpn. J. Appl. Phys., Part 2 35, L1524 (1996).

${ }^{5}$ K. Matsumoto, Proc. IEEE 85, 612 (1997).

${ }^{6}$ E. S. Snow, P. M. Campbell, F. A. Buot, D. Park, C. R. K. Marrian, and R. Magno, Appl. Phys. Lett. 72, 3071 (1998).

${ }^{7}$ G. Abadal, A. Boisen, Z. J. Davis, O. Hansen, and F. Grey, Appl. Phys. Lett. 74, 3206 (1999).

${ }^{8}$ F. S. Cien, C.-L. Wu, Y.-C. Chou, T. T. Chen, S. Gwo, and W.-F. Hsieh, Appl. Phys. Lett. 75, 2429 (1999).

${ }^{9}$ S. C. Minne, J. D. Adams, G. Yaralioglu, S. R. Manalis, A. Atalar, and C. F. Quate, Appl. Phys. Lett. 73, 1742 (1998).

${ }^{10}$ R. García, M. Calleja, and F. Pérez-Murano, Appl. Phys. Lett. 72, 2295 (1998).

${ }^{11}$ R. García, M. Calleja, and H. Rohrer, J. Appl. Phys. 86, 1898 (1999).

${ }^{12}$ K. Morimoto, K. Araki, K. Yamashita, and M. Niwa, Appl. Surf. Sci. 117/118, 652 (1997).

${ }^{13}$ P. Avouris, T. Hertel, and R. Martel, Appl. Phys. Lett. 71, 285 (1997).

${ }^{14}$ P. A. Fontaine, E. Dubois, and D. Stievenard, J. Appl. Phys. 84, 1776 (1998).

${ }^{15}$ T. Teuschler, K. Mahr, S. Miyazaki, M. Hundhausen, and L. Levy, Appl. Phys. Lett. 67, 3144 (1995).

${ }^{16}$ J. A. Dagata, T. Inoue, J. Itoh, K. Matsumoto, and H. Yokoyama, J. Appl. Phys. 84, 6891 (1998).

${ }^{17}$ F. Pérez-Murano, K. Birkelund, G. Abadal, K. Morimoto, and J. Dagata, Appl. Phys. Lett. 75, 199 (1999).

${ }^{18}$ M. Calleja, J. Anguita, R. García, K. Birkelund, F. Pérez-Murano, and J. Dagata, Nanotechnology 1, 10 (1999).

${ }^{19}$ F. Marchi, V. Bouchiat, H. Dallaporta, V. Safarov, D. Tonneau, and D. Poppelt, J. Vac. Sci. Technol. B 16, 1 (1998).

${ }^{20}$ R. García and A. San Paulo, Phys. Rev. B 60, 4961 (1999).

${ }^{21}$ E. S. Snow, P. M. Campbell, and F. K. Perkins, Appl. Phys. Lett. 75, 1476 (1999).

${ }^{22}$ The value of $D=10^{-9} \mathrm{~cm}^{2} \mathrm{~s}^{-1}$, has been interpolate from the diffusion constant of $\mathrm{OH}^{-}$in water $\left(\sim 5.3 \times 10^{-5} \mathrm{~cm}^{2} \mathrm{~s}^{-1}\right.$, CRC Handbook of Chemistry and Physics (CRC, Boca Raton, FL, 1994), and the diffusion constants of several atoms on silicon $\left(\sim 10^{-14}-10^{-20}\right)$ [see M. ZinkeAllmang and L. C. Feldman, Appl. Surf. Sci. 33134, 395 (1988)]. 